\title{
Paroxysmal dysarthria and other transient neurological disturbances in disseminated sclerosis
}

\author{
M. L. E. ESPIR, SYLVIA M. WATKINS, AND HONOR V. SMITH \\ From the Departments of Neurology, Leicester Royal Infirmary and United Oxford Hospitals
}

The alternation of relapse and remission is one of the outstanding characteristics of disseminated sclerosis. Relapse has been defined as 'the appearance of a new symptom . . . or the return or exacerbation of a previous symptom' (McAlpine, Compston, and Lumsden, 1955). Classically, these symptoms last for days, weeks, or months. Distinct from the true relapse, the 'fluctuation' (Müller, 1949), or 'temporary exacerbation' is also well recognized and has been defined as 'the re-appearance of an old symptom or the exaggeration of an existing symptom for a short period, i.e., minutes, hours, or a few days' (McAlpine et al., 1955).

McAlpine subdivides these short-lived attacks into two groups, according to whether they are associated with exogenous factors or occur in paroxysms (McAlpine, Lumsden, and Acheson, 1965). The present study is concerned only with the paroxysmal variety or transient neurological disturbances as they are called here.

Our interest in these disturbances was aroused by six patients, all of whom experienced short-lived, recurrent attacks of dysarthria, accompanied by other symptoms referable to a lesion in the brainstem. In five of the cases, the diagnosis of disseminated sclerosis seems inescapable, whilst in the sixth no firm diagnosis can yet be made. Although Andermann, Cosgrove, Lloyd-Smith, and Walters (1959) described two similar cases from Canada, we can find no similar reports from Great Britain. However, our experience now suggests that such cases, although unusual, are probably commoner than generally realized, and in view of the diagnostic difficulties we are presenting our cases here. In doing so, we particularly hope that other workers will more readily recognize this type of transient neurological disturbance and prepare the systematic studies required for the elucidation of the aetiological problems.
CASE 1 (L.R.I. NO. 333176)

A dental nurse, aged 24, was first seen in June 1962 complaining of short-lived, recurrent attacks in which she saw double and had difficulty in talking, swallowing, and walking.

Four months earlier she had been troubled by a constant 'dizziness' and her left arm and leg became clumsy. This clumsiness only lasted a few days, though the dizziness persisted for about six weeks.

For the next four weeks she felt perfectly well. The attacks then began and became steadily more frequent until, by the time of her visit to the clinic, she was having from three to four every hour throughout the day. They were ushered in by a warning sensation of 'dullness', quickly followed by diplopia, by difficulty in pronouncing, but not in finding words, by difficulty in swallowing, and by stiffness and unsteadiness of the legs. She described how, if she felt the warning 'dullness' when walking in the town, she would stop and pretend to be looking in a shop window so as not to attract the attention of the passers-by who, she felt sure, would conclude from her gait and her speech that she was drunk. The attacks lasted for only a few seconds and between them she had no symptoms whatever. She said that they were often brought on by stress or emotion.

During her visit to the out-patient clinic she did not have an attack. Examination revealed slight pallor of the left optic disc, nystagmus on lateral gaze, mild incoordination of both hands, some increase in the knee and ankle jerks, and bilateral extensor plantar responses. Her speech was quite normal. A tentative diagnosis of disseminated sclerosis was made, and three weeks later she was admitted to hospital.

Except that she now had mild but definite dysarthria even between the attacks, her physical condition was unchanged. The attacks were as frequent as ever and several were witnessed. The patient would suddenly become grossly dysarthric and ataxic, and if standing, would catch at some support to prevent herself from falling. She invariably recovered within a few seconds, too quickly in fact to allow any formal neurological examination. Her conscious level was unaffected. 
Routine blood tests, and the cerebrospinal fluid pressure and constituents were normal, and the Wassermann reaction was negative. Plain radiography of skull and chest and vertebral angiography showed no abnormality. The electroencephalogram (E.E.G.) showed widespread medium theta activity over both hemispheres, and overbreathing provoked several paroxysms of bilateral, synchronous, high-voltage, 3 per second slow waves. She had no attacks during this examination.

She was treated with a course of adrenocorticotrophic hormone (A.C.T.H.). Sixty units were given twice daily by intramuscular injection for a week and thereafter the dose was gradually reduced. During this time the frequency of the attacks steadily fell from between $\mathbf{4 0}$ and 50 daily to only one a day. The physical signs also improved until, by the time she was discharged after two weeks in hospital, they were limited to bilateral extensor plantar responses and slight difficulty in writing.

For the next six months she was very well but then, in January 1963, she had a heavy cold and, at the same time, the attacks began again. Their frequency steadily increased until, at the end of six to eight weeks she was having as many as 80 a day. The pattern and duration of attacks were unaltered.

In March 1963 she was readmitted to hospital. The physical findings were still unchanged and, once again, the cerebrospinal fluid was normal. This time she was treated with phenytoin, $100 \mathrm{mg}$. three times daily, and primidone, $125 \mathrm{mg}$. three times daily. By the end of a fortnight of this regime the attacks had stopped, though she would still occasionally experience the premonitory 'dull' feeling. She was discharged after two weeks in hospital. In April the primidone was withdrawn because she developed a rash, and shortly afterwards the phenytoin was also withdrawn as it was making her sleepy. Within a few days the attacks began again, although at the greatly reduced frequency of one every two or three days. Phenytoin was again prescribed, this time in combination with dexamphetamine, and again the attacks stopped completely.

Since that time she has been seen at regular intervals. In January 1964 she had another bout of persistent diplopia, which lasted for about two weeks. When she was last seen, in June 1965, she had a trace of dysarthria, diplopia on looking to the right, and occasionally tended to lose her balance. Otherwise she had remained well.

Here, both the evolution of the illness, with the clear-cut relapses and remissions, and the physical findings of cerebellar and pyramidal tract signs and pallor of one optic disc, are characteristic of disseminated sclerosis. Both the second and third relapses consisted of repeated paroxysms of brain-stem disturbance and especially dysarthria. The complete dissociation between these paroxysms and the E.E.G. changes does not support an epileptic basis, and the investigations gave no evidence of any other lesion, such as an angioma of the brain-stem. It would seem, therefore, that the paroxysmal dysarthria was a manifestation of the disseminated sclerosis. We were unfamiliar with this possibility at the time, and in studying this, our first case, we were unaware that overbreathing might prove a precipitating factor. No attempt was therefore made to study this effect, except incidentally when overbreathing during the E.E.G. examination. Similarly other factors, such as the effect of alcohol and vasodilator agents were not tried, but during her third relapse the inhibitory effect of phenytoin with dexamphetamine was unmistakable. It is also of interest that the patient herself had noticed that emotional stress aggravated the attacks, and this was also a characteristic of most of our later cases.

In the second case, our interest in the effect of overbreathing was aroused when this was seen to precipitate attacks during the E.E.G. examination. As in the previous case, the paroxysmal dysarthria also occurred early in the course of the illness.

CASE 2 (L.R.I. NO. 343317)

A housewife, aged 40, was admitted to hospital in November 1962, complaining of attacks of difficulty in speaking.

Four months earlier she had diplopia, which lasted for four days. She was then quite well until November 1962,0 when she began to have frequency of micturition. A fewD days later she became feverish and complained of dysuria $\stackrel{\rho}{\mathrm{D}}$. Her general practitioner treated her with sulphonamideso and her urinary symptoms subsided. While she was stile feverish, however, the difficulty with speech began. Sheo described attacks of slowness and slurring of speech, which she said was preceded by a 'warning feeling in the head'. The attacks lasted up to 15 seconds, and recurreds once or twice every hour. There were no other symptoms at this time. Except for a previous attack of 'cystitis' in 1955, the past and family histories were irrelevant. As soon as an attack was witnessed, it was clear that the disturbance of speech was, in fact, dysarthria. Examination revealed no abnormalities other than the attacks themselves, and between them her speech was normal.

On investigation, the blood picture and cerebrospinal fluid proved normal, as did radiography of the chest and skull. The erythrocyte sedimentation rate (E.S.R.) was greatly raised, presumably because of a persistent urinary infection with $E$. coli. The E.E.G. showed low voltage alpha activity at 10 to $11 \mathrm{c} / \mathrm{s}$ and fast activity at 22 to $26 \mathrm{c} / \mathrm{s}$ extending from the frontal regions backwards; occasional low voltage theta activity at 4 to $6 \mathrm{c} / \mathrm{s}$ occurred in both post-central areas, being more marked on the left than the right, and increased by overbreathing. She had no attacks during this recording. Left carotid arteriography showed no abnormality. Prostigmine was then tried, but had no effect.

The frequency of paroxysms slowly increased to about 30 per day, and their pattern became more complex in that the dysarthria was now accompanied by blurring of vision and clumsiness of the hands and legs. In spite of this, a second E.E.G., taken two months after the first, showed that the earlier abnormalities had disappeared. The frequency of the attacks then began to diminish 
and when, in March 1963, she began taking phenytoin, $50 \mathrm{mg}$. three times daily, it fell further, to between 0 and three daily.

After a month of this treatment, she suddenly noticed a pricking sensation in the left fingers and cheek, and that the left foot was dragging. This lasted for three weeks and then cleared completely. About a week after the onset of these symptoms, the attacks of dysarthria increased in frequency. The phenytoin was then abandoned, and later primidone was substituted.

She was readmitted on 21 May 1963. Re-examination now showed nystagmus in all directions with diplopia on lateral gaze. The only other abnormality was slight incoordination of the left arm. The diagnosis of disseminated sclerosis seemed increasingly probable, and further examination of the cerebrospinal fluid showed a change in the Lange curve to 22110000 , although the other constituents were again normal. The blood picture and E.S.R. were also normal. A third E.E.G. in May 1963 again showed low-voltage theta activity, mainly in the left post-central area. When overbreathing she had four attacks of slurred speech with tingling in the right arm, face, and leg. There was, however, no change in the record during these attacks.

She was discharged on 23 May free from attacks. However, on 26 May the attacks began again and now the dysarthria was accompanied by diplopia, paraesthesiae in the tongue, and incoordination of the left arm and leg. They occurred about three times per hour and, as before, lasted for a few seconds only. A few days later the right leg began to drag and the right arm grew clumsy. In addition, for the first time, the dysarthria persisted between attacks and was accompanied by paraesthesiae of the left side of her face and diplopia. She was therefore, readmitted to hospital on 10 July.

Examination now showed marked dysarthria and gross nystagmus with diplopia in all directions. Tone and power in the limbs were normal, but coordination was poor in the right arm and her gait was very unsteady. The jaw jerk and arm reflexes were extremely brisk, the left abdominal reflexes were reduced, and the right plantar response extensor. There was no sensory disturbance. In the cerebrospinal fluid, the Lange curve was now frankly paretic (43210000), and the gamma globulin level was increased to $28.2 \%$ of the total protein. The Wassermann reaction was negative.

She was treated with A.C.T.H. by intramuscular injections of 60 units twice daily, with gradual reduction of the dose over the course of 10 days. She improved rapidly, and when discharged on 23 July her gait and the coordination of her right arm and leg were almost normal. Her speech was now normal and the attacks had stopped completely.

Five months later, she was free from all symptoms and playing golf as well as ever. She had no further attacks.

When this patient was first seen, the recurrent paroxysms of dysarthria were the only manifestation of what ultimately proved to be disseminated sclerosis. Indeed, it was only the evolution of the illness, which included the development of the typical abnormality of the Lange curve, that put the diagnosis beyond reasonable doubt. As in case 1, there is no evidence that the paroxysms were epileptic. The effect of phenytoin was much less definite than in the previous case nor was emotional stress noted as a precipitating factor. By the time of the third E.E.G., however, attacks could definitely be precipitated by overbreathing.

$$
\text { CASE } 3 \text { (L.R.I. NO. 351420) }
$$

A housewife, aged 24, was first seen in March 1963, complaining of attacks of slurred speech, pins and needles in the right arm and leg, and difficulty in controlling the right arm.

Ten months earlier, in May 1962, she had an episode of diplopia and blurred vision lasting for two weeks. The following September she had dysphonia for two days. She said that on waking one morning she had found she could only whisper, although she was able to say all she wanted. There was no other symptom whatever. During the next month, she had 10 days of urgency and frequency of micturition, followed by dysuria. She was then well until February 1963, when the right side of her face became numb and tingled, while at the same time she found difficulty in keeping her balance. These symptoms cleared after three weeks, and it was about a week later that the attacks began.

These attacks lasted for a few seconds only and recurred every few minutes. In them, her speech became slurred; she knew what she wanted to say but could not move her mouth to pronounce the words properly. At the same time her legs became stiff and unsteady. She also described a feeling of 'woolliness' of the head, but she had no difficulty in thinking or understanding during the attacks, and they did not interfere with her reading, nor with watching television. Between the attacks she felt perfectly normal, but at one time they recurred so frequently that they seriously interfered with her housework since she had constantly to stop for a moment or two until the attack passed off. She thought they were worse after having a hot bath. During the next three weeks, the frequency of the attacks decreased spontaneously to about three a day or less.

On examination in March 1963, she had ataxic nystagmus, minimal ataxia of both legs, and impaired vibration sense in the right leg. The optic discs were normal and both plantar responses flexor, though the lower abdominal reflexes were absent.

It was thought that she probably had disseminated sclerosis and, in April 1963 she was admitted to hospital. On re-examination then, the only abnormality found was impaired vibration sense in the right arm and leg. The blood picture, cerebrospinal fluid, and radiography of the skull and chest were normal. Further examinations in May and July 1963 again showed ataxic nystagmus, although she had not had further attacks or other symptoms. By June 1965 she had had no further trouble.

Except for the paroxysmal dysarthria the history in this case is fairly typical of the early stages of 
disseminated sclerosis. In view of the experience gained with cases 1 and 2 , the bout of paroxysmal dysarthria and ataxia was interpreted as one relapse which remitted spontaneously over the course of about one month.

In all these three cases the paroxysmal dysarthria occurred early in the course of the disease and, as previously we were unaware that this might be a manifestation of disseminated sclerosis, it was sufficiently bizarre to make the diagnosis difficult. However, in the fourth case paroxysmal dysarthria appeared as part of an unmistakable relapse, late in the illness, in a case in which the diagnosis was already firmly established.

\section{CASE 4 (OXFORD D.S. NO. 99)}

A woman of 30 was first seen on 3 September 1957. Two years before, early in her first pregnancy, she had had an attack of retrobulbar neuritis. This soon recovered and, in July 1956, she was delivered of a healthy baby. Two months later, after some unaccustomed exertion, she noticed a burning sensation in both knees and her right leg dragged badly enough to interfere with her walking. She also developed tingling paraesthesiae in the fingers of both hands. She had had a little frequency and precipitancy of micturition ever since the birth of the baby. The symptoms in the legs and hands slowly improved and by May 1957 she seemed to have recovered. Four months later, however, in September 1957, both legs became heavy and weak, and the paraesthesiae of the hands returned.

Examination at this time revealed pallor of the right optic disc, nystagmus on lateral gaze to both sides with some cerebellar incoordination of all limbs. In addition, the deep tendon reflexes were increased, especially on the right; there was ill-sustained, bilateral ankle clonus, but the plantar responses were still flexor. The diagnosis of disseminated sclerosis was made and her name was put on the waiting-list for admission to hospital.

She was admitted on 2 January 1958. Since her visit to the out-patient clinic she had improved, although she was still not walking normally. On examination, the nystagmus and ataxia were still detectable, but the tendon reflexes were now within normal limits. The abdominal reflexes, however, could not be obtained, the right plantar response was now equivocal and the left probably flexor. Lumbar puncture showed clear cerebrospinal fluid with normal manometry, 4 cells per cubic millimetre, protein $52 \mathrm{mg}$. per $100 \mathrm{ml}$., Lange 4455432110, and Wassermann reaction negative. She was treated with intrathecal injections of tuberculin (Smith, Espir, Whitty, and Russell, 1957). She showed considerable tolerance to tuberculin and the reaction in the cerebrospinal fluid was of the type seen in a minority of cases of disseminated sclerosis (Smith, Hughes, and Hunter, 1961). She quickly recovered from the reactions and when discharged on the 20 February 1958, was walking normally and had full control of her bladder.

Since that time she has been seen at regular intervals. During the next year she occasionally complained that her legs had felt stiff or that she had again become aware of the paraesthesiae in the fingers. These attacks lasted for only a few days and were always associated with some such event as a heavy cold or, once, an accidental fall, while the physical signs remained unchanged. They appeared, therefore, to be in the nature of temporary exacerbations. In February 1959, however, she suffered an unmistakable relapse. Following an attack of influenza she developed vertigo and nausea, the frequency and precipitancy of micturition recurred, and the nystagmus and ataxia of her hands reappeared. Two months later the left arm and leg became a little weak.

During the late summer of 1959 she again improved, but in January 1960 she had another relapse in which she developed a moderately severe spastic paraparesis. Since January 1961 this was steadily progressive and by 1962 she was virtually chairbound.

In August 1961 she had a recurrence of the vertigo and nausea that had troubled her early in 1959, but this time it was much worse. It occurred in increasingly frequent attacks and made walking and even standing very difficult. It was unaffected by prochlorperazine. After six months it began to remit and finally stopped altogether.

There were no further neurological episodes until August 1964 when the vision in both eyes became blurred and then slowly improved. At the same time, the attacks of vertigo began again, but only lasted a few weeks before they were largely superseded by a different type of attack consisting of stiffness and weakness of the right arm and@ difficulty in speaking. These attacks were, she claimed $\mathbb{D}$ precipitated by emotional stress. One day, when she was alone in the house, she was called on by someone she did not know. On trying to speak to this stranger, she was greatly embarrassed to find that her speech became a meaningless jumble while, at the same time, her righto arm became stiff and 'jerked'. The whole attack was over? in less than a minute, but it proved to be the first of a long series that lasted until the end of 1964.

Many of these attacks were witnessed. They occurred spontaneously at least once every 30 minutes, and lasted from 30 to 40 seconds. She would be talking normally when her speech would become slurred and incomprehensible. None of the observers had any doubt that the difficulty was one of articulation rather than of finding words; that they were, in fact, paroxysms of dysarthria and not of dysphasia. At the same time, movements of the right hand became slow and stiff, and incoordinate. This was the 'jerking' she had complained of. There was no alteration in consciousness, colour, or pulse rate.

The attacks could be brought on at will by asking the patient to overbreathe. After as few as five or six forced expirations she would say, 'Look, it's happening'; and for the next 30 seconds her speech was incomprehensible and the right hand became stiff. When overbreathing into a plastic bag, no attacks were produced. A small quantity of alcohol ( $2 \mathrm{oz}$. of sherry) seemed to raise the threshold, in that 12-15 forced expirations were then required to bring on an attack. Phenytoin had no appreciable effect on the frequency of the attacks.

Several attacks were provoked by overbreathing under electroencephalographic control. The resting record was normal. Ten to 20 seconds of overbreathing regularly 
produced brief episodes of dysarthria during which there was some movement artefact, but no electroencephalographic change. Electromyography (E.M.G.) was also carried out by Dr. Geoffrey Rushworth and these studies showed that the movements of the right hand, provoked by overbreathing, were due to a marked exacerbation of the cerebellar incoordination.

As the weeks passed, the attacks of dysarthria became less frequent until, by the end of November 1964, they had stopped and could no longer be provoked by overbreathing. When she was last seen, in March 1965, her condition was much as it had been before the severe relapse of the previous August, except that the right hand was probably a little weaker.

In this case, the unequivocal evidence of multiple lesions, including retrobulbar neuritis, together with the well-defined relapsing-remitting course and findings in the cerebrospinal fluid, make the diagnosis of disseminated sclerosis inescapable. Here, the paroxysms of dysarthria were a relatively late phenomenon. As in all the cases, however, they were clearly related to a relapse. Their regularity was such that it was possible to study the effects of overbreathing, rebreathing, and alcohol on their frequency, and on the E.E.G. and E.M.G.

\section{CASE 5 (L.R.I. NO. 396724)}

A plumber, aged 43 years, was first seen on 2 June 1965 . Three months earlier he had had an influenza-like illness, and began to see double. This diplopia lasted for a week and then cleared completely. Shortly afterwards he began to have attacks of difficulty in speaking, in using his hands and in walking. There was no other relevant medical or family history. These attacks only lasted for a few seconds but recurred several times during the day. His speech became slurred, his hands grew so clumsy he could not use his tools and, if walking, he would stagger so that he had to hold on to some support until his balance returned. They were brought on by emotional stress and by physical exertion; thus even walking 200 yards was usually enough to precipitate an attack.

When he was first seen the difficulty in speech was immediately apparent as simply coming into the room precipitated an attack. He became so dysarthric that he was scarcely intelligible and at the same time was obviously ataxic. The attack was over so soon that there was no time for any formal examination. Between attacks examination revealed pallor of both optic discs, a generalized increase in the deep tendon reflexes especially on the right side, and an equivocal right plantar response. As in case 4, a short period of voluntary overbreathing invariably precipitated an attack of dysarthria and ataxia. Both speech and coordination reverted to normal after about 10 seconds.

He was admitted to hospital for investigation but with negative results. In particular, lumbar puncture yielded clear colourless cerebrospinal fluid which was normal on manometry and on analysis. Radiography of skull, cervical spine and chest, and arch aortography showed no abnormality. While in hospital he had a few attacks every day. Tegretol, $100 \mathrm{mg}$. t.d.s., was prescribed and the attacks stopped, and did not recur when the tegretol was withdrawn a week later. When last seen in November 1965 , he was free from symptoms and back at work.

This patient's general practitioner had been so impressed by the combination of the difficulty in speech and balance with the history of diplopia that he had referred him with a provisional diagnosis of disseminated sclerosis. Although so far, at least, the dysarthria and incoordination have occurred only in brief paroxysms, the physical findings, negative investigations and, above all, the close resemblance of the attacks to those seen in our other cases strongly suggest that this is, indeed, the correct diagnosis.

In the next case there has been no neurological episode other than the paroxysms themselves. However, the resemblance between these paroxysms and those in the previous cases is sufficiently striking to warrant a short description.

CASE 6 (L.R.I. NO. 152158)

A publican, aged 40 years, was first seen in April 1965. Three months before he had begun to have brief, recurrent attacks of difficulty in speaking. By the time he was seen he was having between 40 and 50 attacks in the day. In each attack, which lasted about 10 seconds, his speech became slurred and his legs unsteady. Sometimes he also saw double, and this might outlast the other symptoms by as much as two minutes. He had noticed that the attacks were more frequent when he was worried and that they might be brought on by turning his head suddenly.

On examination there were no abnormal findings other than the attacks themselves. These consisted of sudden paroxysms of gross dysarthria and moderate ataxia and were too brief to allow of any formal examination. As in the last two cases they could be brought on at will by asking the patient to overbreathe.

Shortly after he was first seen the frequency of the attacks began to abate until he was virtually free from them. However, after an interval of about two weeks, they began again and this time recurred every two to three minutes. Their character was unchanged and, as before, they were always worse when he was emotionally upset. He had also developed a new symptom in that, distinct from the paroxysmal dysarthria, he had transient attacks of burning paraesthesiae in the right side of his face and right hand. These also only lasted a few seconds and recurred five to six times in the hour.

He was treated with phenytoin, $100 \mathrm{mg}$. t.d.s., and the attacks of dysarthria, diplopia, and ataxia at first improved but later returned as frequently as ever. In May 1965 he was admitted to hospital. Physical examination showed no abnormality other than the attacks themselves. The blood and cerebrospinal fluid were both normal and the Wassermann reaction was negative. Radiography of 
skull and chest was normal, the cervical spine showed narrowing of the 5/6 disc space, and arch aortography showed no abnormality.

Tegretol, 100 mg. t.d.s., was substituted for the phenytoin and the attacks subsided. When last seen in October 1965 he was free from symptoms and back at work.

So far, all the symptoms here can be ascribed to a single lesion in the brain-stem. Accordingly, no firm diagnosis of disseminated sclerosis can be made. The paroxysms of dysarthria were, however, quite remarkably like those in our other cases, while investigation revealed nothing to suggest an alternative diagnosis. Moreover, transient neurological disturbances are not infrequently among the earliest symptoms of disseminated sclerosis (McAlpine, 1955). This diagnosis must therefore remain a distinct possibility.

\section{DISCUSSION}

These cases illustrate that recurrent paroxysmal attacks of dysarthria accompanied by other signs referable to a lesion in the brain-stem, may be interspersed between, or even precede, the more typical symptoms of disseminated sclerosis. In the first five cases, we consider that there can be little doubt that the bouts of paroxysmal dysarthria were, in fact, due to disseminated sclerosis. Although in the absence of any specific test, this diagnosis cannot be made when the paroxysms have been the only manifestation, as in case 6 , nevertheless, in such cases the possibility must be borne in mind.

Such frequently recurring paroxysmal attacks of dysarthria are undoubtedly rare in disseminated sclerosis, but there are many other varieties of transient neurological disturbance which may well have the same significance. According to McAlpine et al. (1955), fleeting episodes are not uncommon. Transient motor, sensory, and visual disturbances have all been described (Franklin and Brickner, 1947; Brickner, 1950; McAlpine et al., 1965), as have trigeminal neuralgia, (Harris, 1950), tonic spasms of the limbs (Matthews, 1958; Zeldowicz, 1961; Joynt and Green, 1962; Lance, 1963), and epilepsy (Wilson and MacBride, 1925; Drake and Macrae, 1961; Matthews, 1962; Ashworth and Emery, 1963).

In a study specially designed to assess the variety and incidence of transient neurological disturbance in disseminated sclerosis, we have seen examples of all the above types, referable to the different parts of the neuro-axis. The transient sensory and visual disturbances were much the commonest, and trigeminal neuralgia mercifully rare. The incidence of transient neurological disturbance of all types was $46 \%$ in a series of 100 consecutive patients with disseminated sclerosis (diagnosed according to strict criteria) compared with only $7 \%$ in a similar series of healthy controls matched for age and sex.

If the definition of relapse quoted in the introduction is accepted, then the true relapse may be considered as the clinical counterpart of the formation of a new plaque or the extension, or reactivation, of an old one. Similarly, the remission corresponds to quiescence of the demyelinating process. Clearly, such transient neurological disturbances as the paroxysmal dysarthria described here come and go far too quickly to be explained on the same pathological basis; they must reflect, rather, some rapidly reversible change. The distinction between relapse and transient neurological disturbance is well seen in trigeminal neuralgia; each separate stab of pain, lasting a matter of seconds, is a transient disturbance while the whole bout, lasting as it commonly does for weeks or months, is a relapse. What exactly happens within the plaque to set up the transient disturbance is not known, but in the hope of throwing some light on this problem we have looked to see what features the attacks of paroxysmal dysarthria in our cases possess in common.

In all the first five cases, and indeed in the sixthe also if the provisional diagnosis ultimately proves to be correct, the attacks began during an active phase of the disease. Either they were a feature of an early episode or, as in case 4 in which they occurred late in the illness, they were accompanied by other unmistakable signs of relapse. In the two cases reported by Andermann et al. (1959) the paroxysmas dysarthria was also an early phenomenon and remitted. In all our cases, too, the attacks ultimately stopped and the patients were left either symptom free, or with some degree of persistent dysarthria. It seems, therefore, that in these cases, at least, the bouts of paroxysms were related to active formation or extension of a plaque rather than to the residual glial scar. Whether this is true of the paroxysmal variety of transient neurological disturbance as a whole we do not know. It is, however, interesting that the tonic motor seizures reported both by Matthews (1958) and Zeldowicz (1961) seem to have followed much the same pattern.

Another striking feature was the relationship of the attacks to emotional stress and to overbreathing. Two patients made no mention of the effect of emotion; unfortunately they were not specifically questioned on this point. The other four all volunteered the information that any emotional stress would precipitate an attack and several demonstrated this by having one under the embarrassment of being interviewed. Four patients invariably had an attack when they were required to overbreathe; indeed the regularity and rapidity with which an 
attack developed was remarkable. In case 4 , for example, the frequency of the attacks was already waning when the effect of overbreathing was observed. Yet six or seven forced expirations were all that were required to render the patient's speech incomprehensible. After taking a little alcohol, a longer period of overbreathing was necessary to bring on an attack, while if she rebreathed from a plastic bag there was no attack.

In view of these findings, we venture to advance the following hypothesis as to the mechanism of these paroxysmal attacks. The fact that the attacks are an early symptom suggests that they may correspond to the earlier stages of demyelination, to the formation, for example, of the 'shadow' or 'halo' plaque. During this phase it may well be that the axons, though still capable of functioning normally in the various facilitating and inhibiting systems, become very vulnerable to changes in the internal environment and that some minor, and reversible, change might cause them temporarily to suspend function, which they resume as the change passes off. From this stage one of two things might happen: either the early demyelinating process might stop and stability of function return, or it might progress to a further stage causing sustained loss of function. This would agree very well with the pattern of events seen on our cases.

As to the nature of the change, there may, of course, be several factors at work; at least one, however, appears to be highly sensitive to the effects of overbreathing. Since overbreathing is known to decrease cerebral blood flow, it is tempting to postulate that the factor concerned in our cases was a small fall in the blood supply producing, presumably, a minor degree of hypoxia, to which damaged neurones are abnormally sensitive. The disorders of function may thus result from a degree of hypoxia which is too small to have any detectable effect on normal neurones. This, too, accords well with our clinical observations, in that overbreathing was no longer able to produce attacks once these had remitted. That hypoxia can produce recurrent transient neurological disturbances is supported by the fact that they have been described in rheumatic heart disease and, here too, they are sometimes associated with overbreathing (Hutchinson and Stock, 1963). Whether the same explanation holds for the effect of emotional stress is not clear. It is, however, quite possible that patients who are anxious or embarrassed may alter their respiratory rhythm or rate without being aware of it. The action of the drugs, phenytoin and tegretol, is also of considerable interest. Their use in our cases was prompted by the suggestion that they may act directly on the brainstem, an idea that has been advanced to explain their apparent beneficial effect in some cases of trigeminal neuralgia (Blom, 1962; Spillane, 1964). The response to these drugs in our cases was not constant, but in one the attacks stopped with the administration of phenytoin and in two with tegretol, although in the other cases these drugs were either ineffective or not tried.

We are very conscious that further research on precipitating and relieving factors is needed before the mechanism of transient neurological disturbances can be elucidated. Measures that might usefully be tried include exertion and heating, since one of our patients (case 5) noticed a close relationship between the onset of an attack and a measured amount of exercise, while another (case 3) thought that a hot bath would bring on a paroxysm. Our limited observations with alcohol suggest that vasomotor drugs might also repay study, and other factors, which might impair the local circulation, require consideration, for example, sludging of the solid components of the blood and increased adhesiveness of platelets (Field and Caspary, 1964), and the development of vasospasm in response to stress. The cases we have described here would be particularly suitable for such studies, in view of the frequency and regularity of the paroxysms. We have therefore presented our observations in the hope that other workers encountering similar cases may study some of the biochemical and/or vascular changes responsible for transient neurological disturbances, since a proper understanding of the fundamental processes in disseminated sclerosis must allow for the paroxysmal as well as the more prolonged manifestations of the disease.

\section{SUMMARY}

Five cases of disseminated sclerosis are described, together with a sixth possible case, each having frequent paroxysmal attacks of dysarthria, with or without other symptoms of brain-stem disturbance.

It is concluded that disseminated sclerosis must be considered in the differential diagnosis of cases of paroxysmal dysarthria or other transient neurological disturbances, which are not uncommon either as the presenting manifestation of the disease, or interspersed among longer lasting neurological symptoms.

The pathogenesis of these transient neurological disturbances is discussed. The hypothesis is advanced that some at least may be due to increased susceptibility to hypoxia of nerve fibres in the early stages of demyelination.

S.M.W. wishes to acknowledge a grant towards expenses from the neurological research fund of the Leicester No. 1 Hospital Management Committee. 


\section{REFERENCES}

Andermann, F., Cosgrove, J. B. R., Lloyd-Smith, D., and Walters, A. M. (1959). Paroxysmal dysarthria and ataxia in multiple sclerosis. Neurology (Minneap.), 9, 211-215.

Ashworth, B., and Emery, V. (1963). Cerebral dysrhythmia in disseminated sclerosis. Brain, 86, 173-188.

Blom, S. (1962). Trigeminal neuralgia; its treatment with a new anticonvulsant drug. (G 32883). Lancet, 1, 839-840.

Brickner, R. M. (1950). The significance of localized vasoconstrictions in multiple sclerosis. Res. Publ. Ass. nerv. ment. Dis., 28, 236244.

Drake, W. E., and Macrae, D. (1961). Epilepsy in multiple sclerosis. Neurology (Minneap.), 11, 810-816.

Field, E. J., and Caspary, E. A. (1964). Behaviour of blood platelets in multiple sclerosis. Lancet, 2, 876-880.

Franklin, C. R., and Brickner, R. M. (1947). Vasospasm associated with multiple sclerosis. Arch. Neurol. Psychiat. (Chic.), 58, 125-162.

Harris, W. (1950). Rare forms of paroxysmal trigeminal neuralgia, and their relation to disseminated sclerosis. Brit. med. J., 2,10151019.

Hutchinson, E. C., and Stock, J. P. P. (1963). Paroxysmal cerebral ischaemia in rheumatic heart disease. Lancet, 2, 653-656.

Joynt, R. J., and Green, D. (1962). Tonic seizures as a manifestation of multiple sclerosis. Arch. Neurol. (Chic.), 6, 293-299.
Lance, J. W. (1963). Sporadic and familial varieties of tonic seizures, J. Neurol. Neurosurg. Psychiat., 26, 51-59.

McAlpine, D. (1955). The clinician and the problem of multiple sclerosis. Lancet, 1, 1033-1038.

- (1961). The benign form of multiple sclerosis. Brain, 84, 186-203.

_-, Compston, N. D., and Lumsden, C. E. (1955). Multiple Sclerosis, p. 136. Livingstone, Edinburgh and London.

$\longrightarrow$ Lumsden, C. E., and Acheson, E. D. (1965). Multiple Sclerosis: A Reappraisal, p. 143. Livingstone, Edinburgh and London.

Matthews, W. B. (1958). Tonic seizures in disseminated sclerosis. Brain, 81, 193-206.

(1962). Epilepsy and disseminated sclerosis. Quart. J. Med. 31, 141-155.

Müller, R. (1949). Studies on disseminated sclerosis. Acta med. scand., suppl. 222.

Smith, H. V., Espir, M. L. E., Whitty, C. W. M., and Russell, W. R. (1957) Abnormal immunological reactions in disseminated sclerosis. J. Neurol. Neurosurg. Psychiat., 20, 1-10.

- Hughes, I. E., and Hunter, G. (1961). Intrathecal tuberculin in disseminated sclerosis: the immunological aspects. Ibid., 24, 101-117.

Spillane, J. D. (1964). The treatment of trigeminal neuralgia; Preliminary experience with 'tegretol'. Practitioner, 192, 71-77.

Wilson, S. A. K., and MacBride, H. J. (1925). Epilepsy as a symptom of disseminated sclerosis. J. Neurol. Psychopath., 6, 91-103.

Zeldowicz, L. (1961). Paroxysmal motor episodes as early manifestations of multiple sclerosis. Canad. med. Ass. J., 84, 937-941. 